Journal of Engineering and Applied Sciences 14 (4): 1219-1225, 2019

ISSN: $1816-949 \mathrm{X}$

(C) Medwell Journals, 2019

\title{
Frequency Reuse Optimization for OFDMA Network
}

\author{
Win Adiyansyah Indra, Mohd Saad Hamid, Norfadzlia Binti Mohd Yusof, \\ Nishanthinidevi A/P Jayraman and Herdy Rusnandi \\ Faculty of Engineering Technology, Universiti Teknikal Malaysia Melaka, \\ Hang Tuah Jaya, 76100 Durian Tunggal, Melaka, Malaysia
}

\begin{abstract}
This study investigated the frequency reuse optimization scheme for Orthogonal Frequency Division Multiple Access (OFDMA). Forsk Atoll, Earth Explorer and Global Mapper 15 were used to deploy the simulations, local area (Ayer Keroh, $2.2667^{\circ} \mathrm{N} 102.2833^{\circ} \mathrm{E}$ ) was selected to perform the simulation. The simulation result showed that by implementing Fractional Frequency Reuse (FFR) in Orthogonal Frequency Division Multiple Access (OFDMA) network on the area, gave a significant improvement, in term of co-channel interference and Received Signal Strength Indicator (RSSI). Very low co-channel interference and a strong signal strength occurred on the selected area by modifying the related parameters such as frequency allocation for every single sectoral antenna, antenna height, power of base station and antenna tilt degree. Limited frequency allocation available in the simulation software cause the co-channel interference still exist, although, the values are considered very unsignificant. Improvement can be made by adding frequency resources and physical drive test to validate the simulation measurement result.
\end{abstract}

Key words: Frequence reuse, Orthogonal Frequency Division Multiple Access (OFDMA), co-channel interference, Received Signal Strength Indicator (RSSI), FER, antenna

\section{INTRODUCTION}

Interference is one of the crucial problem in the degradation of the quality of communication over a decade. The major reason behind this problem is due to the limited resource in the radio spectrum and also the number of customers have been increasing dramatically. The quality of the communication is degraded when several users are optimising the same part of the frequencies at the same time and place which is known as interference. Two types of interference which are nature interference and source interference. In nature interference which is known as co-channel interference, the same spectrum used by the two different interfering signals. In adjacent-channel interference, insufficiently separated frequencies using the different interfering signal. In source interference, different user interfacing signals are carried by the same factor is known as intra-cell interference. Inter-cell interference happens when they are carried by different neighbouring portion. In order to overcome this problem, frequency reuse is applied. Frequency reuse is the method of using the same value of the frequency of the radio transmitter within a specific location that are divided by a minimal distance to result in less interference with each other.

The main purpose of this study is to investigate on mitigating the interferences between the frequencies from the adjacent cells and also to increase the network performance by using method of frequency reuse optimization. Due to the higher interference level and increasing traffic demand in metropolitan areas, a better solution has to be found which fulfils the demand of customers. As the number of channels is limited, those channels are reused based on the regular distance intervals. Frequency reuse is very vital in order to gain a beneficial compromise between capacity and performance. As for the access technology of the network that frequency reuse would apply to is Orthogonal Frequency Division Multiple Access (OFDMA).

Frequency reuse patterns: There will be no inter BS (Base Station) macro diversity and no power control is taken up when the frequency resource is globally reused in every cell of the network. Those subscribers at the cell edge eventually have the poor signal strength and sustain the most from inter-cell interference. There are three major frequency reuse patterns for mitigation inter-cell interference which are Hard Frequency Reuse (HFR), Soft Frequency Reuse (SFR) and Fractional Frequency Reuse (FFR).

Hard frequency reuse: Ghaffar and Knopp (2010) described that Hard Frequency Reuse (HFR) separates the bandwidth system into a number of discrete sub-bands

Corresponding Author: Win Adiyansyah Indra, Faculty of Engineering Technology, Universiti Teknikal Malaysia Melaka, Hang Tuah Jaya, 76100 Durian Tunggal, Melaka, Malaysia 


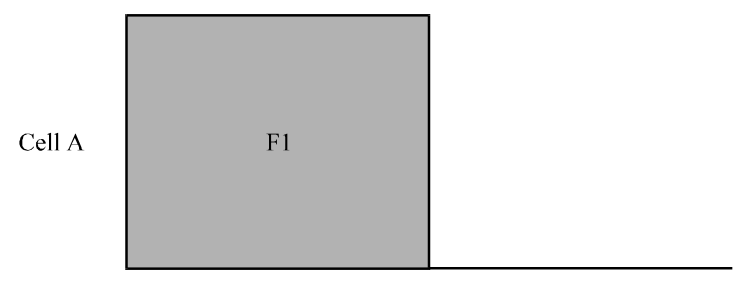

Cell B

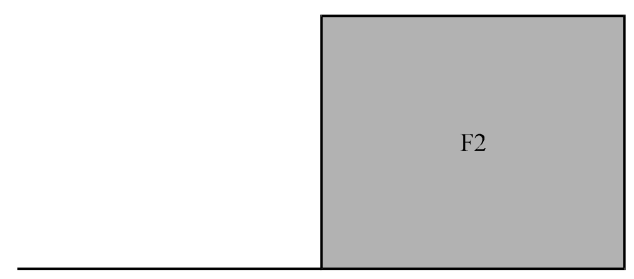

Fig. 1: Hard frequency reuse

based on a selected reuse factor and allow neighbouring cell to transmit on different sub-bands. Hard frequency reuse though simple in implementation suffers from quite reduced spectral efficiency. In HFR, the overall spectrum band is separated into sub-bands F1 and F2 with every sub-band utilised by one of the cells as shown in Fig. 1, HFR fully reject the inter-cell interferences, at the cost of reduced spectrum efficiency.

Mitigation Co-channel Interference (CCI) can be depicted in terms of cell-particular power masks over the bandwidth system. Based on the spectrum portion, the power mask prescribes the fraction of the maximum transmit power that the base station may utilise. The power masks block all but one third of the spectrum in hard frequency reuse with a reuse factor of three (Bohge et al., 2010).

Soft frequency reuse: The Soft Frequency Reuse (SFR) is used to ensure a frequency reuse of one in the cell centre region and a higher frequency reuse factor at the cell edge region. In SFR, the accessible spectrum is split into two sections which are a cell-edge band and cell-centre band. Subscriber within every cell are also split into two parts which are cell-centre subscribers and cell-edge subscribers, according to their geographic area to the BS or other differentiating factors. Cell-centre subscribers can exclusively enter to the cell-centre band and also to the cell-edge band but with lower priority while cell-edge subscribers are limited to the reserved cell-edge band (Bilios et al., 2012).

In SFR frequency designing, a seven-cell hexagonal system layout is used as shown in Fig. 2. (left side) where the cell centre subscribers can utilise the whole frequency band but the cell-edge subscriber's only use partial frequency band non-overlapping with

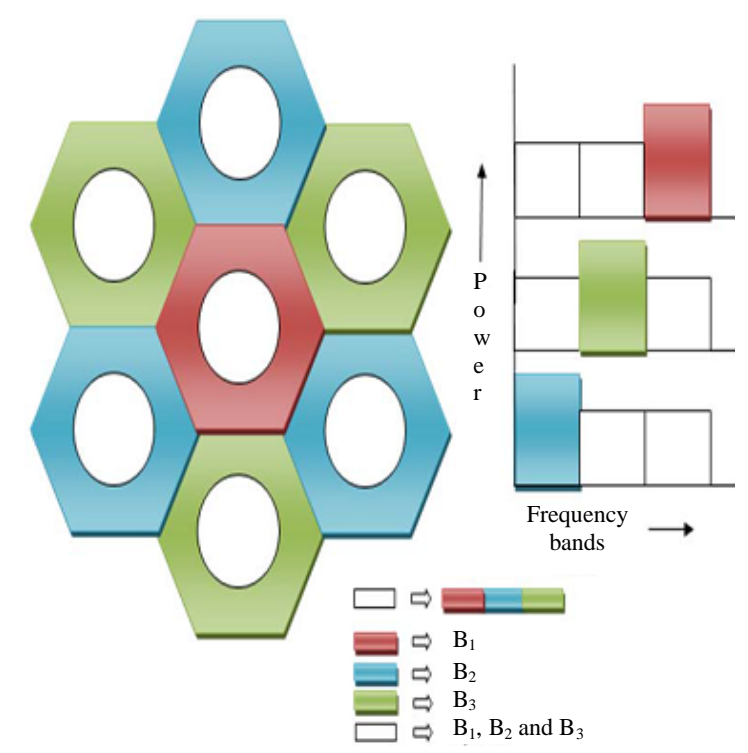

Fig. 2: Power and frequency allocation schemes for soft frequency reuse

neighbouring cells. In SFR, the available spectrum is split into cell-edge and cell-centre band as illustrated in Fig. 2 . (left side). User Equipment (UE) is classified into interior cell and exterior cell UEs based on their position. Cell edge UEs are limited to utilise a part of the available band while cell centre UEs can utilise all usable bands. One third of the available spectrum is allocated to the adjacent cell edges such that for the cell edge traffic have non-overlapping frequency bands and are orthogonal to each other (Bilios et al., 2012).

The cell-centre subscribers can transmit a lower power level while the cell-edge subscriber have to transmit at a higher power level, so that, the data rates can be improved. In Fig. 2 (right side) illustrates the power distribution of SFR. The eNB transmits with a non-uniform power whereas high-power is allotted to UEs which is situated at the cell edge to gain SINR and throughput and low power is allotted to cell-centre UEs. In SFR, the basis of minimising inter-cell interference through planning is classified the subscribers into cell-edge subscribers and cell-centre subscribers based on their geometry factor. For soft frequency reuse, Frequency reuse pattern and frequency reuse factor may be changed according to requirement for coverage, channel capacity and received an interference. The low power subcarriers can be scheduled for users at or close to the cell centre. The high power subcarriers can be scheduled for either cell edge or cell centre users. Power ratio of subcarriers for cell-edge subscribers and subcarriers for cell-centre subscribers are varied (Efnusev et al., 2010). 
Fractional frequency reuse: Fractional Frequency Reuse (FFR) is a mere alternative to the frequency reuse problem in multicellular OFDMA networks. The FFR scheme statically partitions the cell surface into two geographical area which is the inner cell region and the outer cell region close to the cell edge (Mitran and Rosenberg, 2012). FFR highly-developed as a flexible frequency reuse scheme, so that, the performance between spectral efficiency and interference mitigation better compare to the generic frequency reuse.

Frequency reuse is a technique that can increase data throughput of point-to-multipoint systems. In Frequency Reuse 1 system (FR (1)), every cell reuses the overall frequency band to create a possibly high interference. The benefit of using that scheme are to the subscribers who is close to the base-station. It can be seen that a very high rate per unit bandwidth is possible due to the large bandwidth available for those subscribers do not suffer from substantial interference. For subscribers closer to the edge of the cell, substantial interference can cause in low SINR and only a low rate per unit bandwidth is accomplished. Another option is a Frequency Reuse 3 (FR (3)). Every cell use only one third of the system bandwidth, adjacent cells utilise the remaining two thirds with colouring pattern. Cell-edge subscribers endure substantially less interference and accomplish higher rate per unit bandwidth (Chang et al., 2013).

In FR (3), the system bandwidth is split into 3 equal sub-bands where every one of these is allocated to cells, so that, other cell will not utilise the same sub band. Full frequency reuse in every cell can exempt the requirement of advance frequency planning among different cells and the frequency reuse patterns can be adapted on a frame-by-frame basis in every cell. Figure 3 shows an indicative architecture and frequency band allocation. The central BS (Blue Colour), it assumed as high interference is caused by the six direct adjacent. Every cell of the architecture is split into two regions which is inner region and the outer region. The entire available bandwidth of the system is divided into four uneven spectrums (or resource sets), denoted by A (blue), B (green) C (red) and D (yellow). Spectrums A, B, and C have same bandwidth and are allocated in the outer regions with frequency reuse 3. Besides, spectrum D is allocated in all inner regions with frequency reuse 1 . The frequency resources, in all inner regions are utilised as the inner region subscribers are less exposed to inter-cell interference (Ali and Leung, 2009).

The set of subscribers exists in the inner cell region is named as the super group whereas the set of subscribers situated in the outer cell region is known as the regular group. The regular group subscribers are further divided

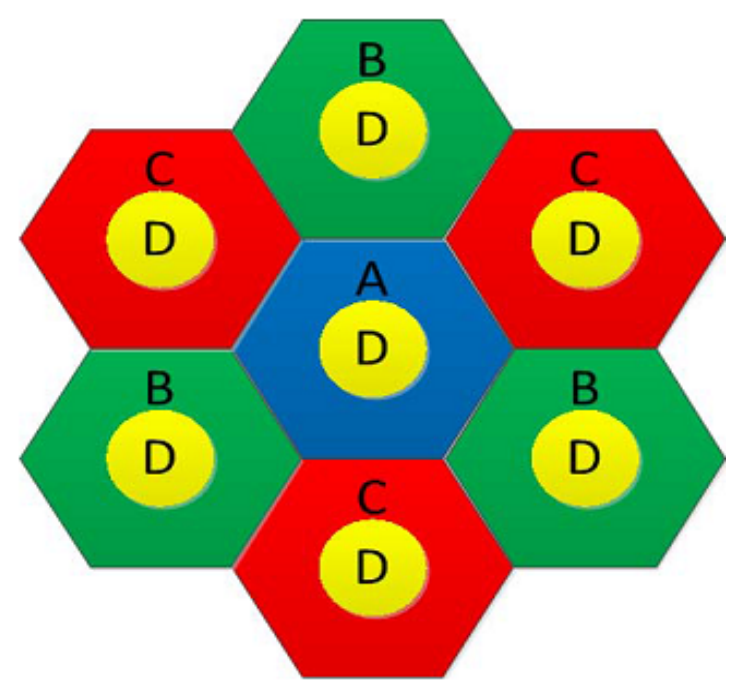

Fig. 3: Proposed frequency band allocation

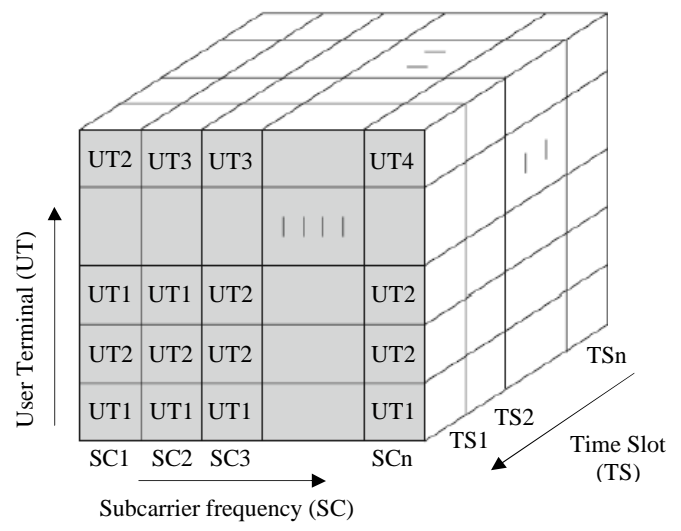

Fig. 4: OFDMA with timeslots and sub-carriers

based on the cell sectors. The gap between the super and regular groups is set according to the basis of the location from the serving BS. The set of subcarriers to serve each group are chosen randomly and their numbers are proportional to the ratio of subscribers in the geographical area to the total number of subscribers in the cell. The set of subcarriers assigned to the groups is reused in the neighbour cells.

OFDMA network: Orthogonal Frequency Division Multiple Access (OFDMA) is a multiuser because subscribers can be classified in both TDMA and FDMA basic where subscriber can be assigned different sub-carriers at different time intervals as shown in Fig. 4 The orthogonality in sub-carriers avoid from multiple access interference.

A single user in OFDMA networks does not need to utilise all the subcarrier at a time. This leads 
to a simultaneous low data rate transmission from different subscribers. Besides, it can be allocated to the non-fading as well as low interference channels for a specific subscriber and avoid poor subcarrier to be assigned. Generally, OFDMA used in most emerging systems such as mobile WiMAX and LTE.

Users on an OFDMA network is specified to a number of sub channels. Those users who are near to the base station allocated with a larger number of channels with a high modulation scheme such as 64 QAM (Quadrature Amplitude Modulation) to send the most data throughput to the respective subscriber. As the subscriber shift further away, the number of sub channels is re-assigned dynamically to fewer and fewer sub channels. The power allotted to each channel is increased. The modulation scheme could step by step move from 16 QAM to Quaternary Phase Shift Keying (QPSK with four channels) and even binary phase shift keying (BPSK with two channels) at longer ranges. The data throughput fall as the channel capacity and modulation varied by maintaining its strength (Bohge et al., 2009).

OFDMA offers flexibility of development across a variety of frequency bands in a system such as LTE and LTE advanced. When it is applied in a cellular system, it is possible to mitigate interference from adjacent cells by utilising the unlike carrier permutations between the two cells or averaged by using allocation with cyclic permutations. OFDMA provide frequency variation by extending the carriers through the used spectrum. A single frequency can be utilise to deliver a good coverage and excellent frequency reuse.

\section{MATERIALS AND METHODS}

Some simulation software tools were deployed to conduct the analysis and optimization of the frequency reuse of selected area. NS-2, OPNET and Atoll were the main softwares used. NS-2 IS an object-oriented, event-driven network simulation tool. It is an open source tool and its main core is based on $\mathrm{C}++$. The programming language used as a front end for simulation configurations is oTCL. NS-2 can be used for protocol design, traffic studies, protocol comparison, etc. A variety of IP networks can be simulated in NS-2. A large number of applications, network elements, network types and traffic models are supported by the tool. Several transport and MAC layer protocols along with different types of traffic sources, queue management mechanisms are also supported. Scenarios based on various telecommunication technologies like General Packet Radio Service (GPRS), Multiprotocol Label Switching (MPLS), AdHoc networks and IPv6 can be implemented and analysed. OPNET is another popular choice for modelling and simulation of communication networks, devices and protocols. It is a commercial tool and is based on an object-oriented modelling approach and graphical editors. Higher OSI layers are the focus of this tool and the lower layers such as the physical layer and the transmission characteristics are modelled quite simply. Although, a special model is available in the OPNET modeller for WiMAX simulations (WiMAX uses OFDMA) which supports multiple cellular networks, multi-sectored base stations, path loss modelling, scheduling for uplink and downlink connections but it is not possible to logically define inner and outer regions in cells. Also, OPNET is not open source, so to additionally include this functionality is not possible. By default, the OPNET modeller supports hexagonal cells and support for irregular cells is not provided. As for the Atoll, Atoll Software was found to be one of the open source simulation tool in which some simulations on frequency reuse is performed. Atoll Software has a separate module for LTE network simulations with some example scenarios as well. Fine control over the base station parameters, their antenna transmission powers and azimuth, allocation of sub-bands to base stations is provided. Also, an important factor in choosing Atoll Software was the simulation of signal strength and frequency plan is easier to conduct.

Another supporting softwares such as Earth Explorer, Global Mapper 15 and Forsk Atoll were used as well. The stated softwares are used at different stages. For Digital Map, the software used for this stage is called Earth Explorer. Earth Explorer is used to explore the world's largest collection of images of the earth's surfaces. The images are in forms such as satellite images, elevation and land cover datasets and digitized maps. The digital maps of earth explorer in the format of Digital Raster Graphics (DRGs). A Digital Raster Graphic (DRG) is used to scan image standard series topographic map of a USGS, by including information all map collar. The image inside the map neat line is geo referenced to the surface of the earth which fit to projection of the Universal Transverse Mercator (UTM). Since, the Earth Explorer Software is an online software, therefore, we need the internet connection in order to get the map. In order to download digital map, we can use software called Global Mapper 15. The last and main software is Forsk Atoll, Forsk Atoll is a radio planning and optimisation software which can run network cell planning where it imported geo data map and all the cellular parameters, like antenna height, location, power, distance, etc., were simulated.

\section{RESULTS AND DISCUSSION}

The selected area for this frequency reuse optimization is surrounding Ayer Keroh, Melaka, Malaysia area $\left(2.2667^{\circ} \mathrm{N} 102.2833^{\circ} \mathrm{E}\right)$. In the DATA window, prediction was made to identify the signal strength of the location. Then, the channel interference of 


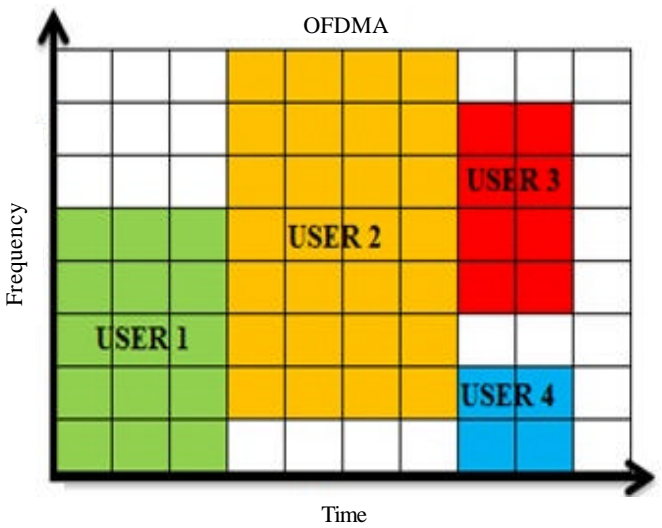

Fig. 5: OFDMA illustration graphic

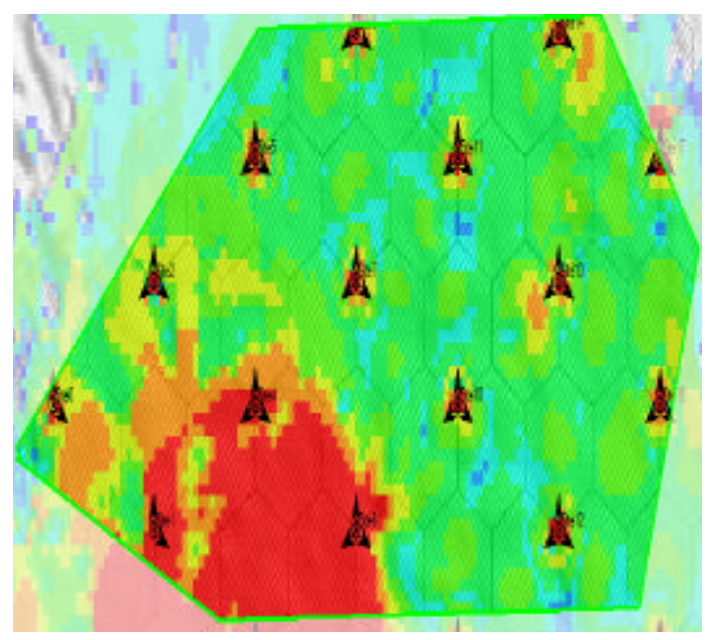

Fig. 6: Signal strength in Ayer Keroh area

downlink was identify by using the prediction also. Downlink is chosen OFDMA because in LTE, OFDMA Network is downlink.

Based on the results in Fig. 5 and 6 can be observed the signal strength is poor and there are large number of co-channel interference. In order to obtain with a strong signal strength and lower number of co-channel interference in a cell each site allocate with different frequencies, the height of antenna is adjusted based on location and finally the maximum power of antenna is adjusted from the range $80-20 \mathrm{dBm}$.

After adjusting the parameters, the prediction for both the signal strength and co-channel interference is run. The output results is shown above. After obtain the Signal strength and Interference prediction, the frequency plan is obtained. By using the transmitter in the DATA window, the frequency plan is calculate automatically. Table 3 shows the interfered cells and the number of
Table 1: Legend of signal strength coverage

\begin{tabular}{|l|l|}
\hline Colors & Legends \\
\hline & Best signal level $(\mathrm{dBm}) \geq-70$ \\
\hline & Best signal level $(\mathrm{dBm}) \geq-75$ \\
\hline & Best signal level $(\mathrm{dBm}) \geq-80$ \\
\hline & Best signal level $(\mathrm{dBm}) \geq-85$ \\
\hline & Best signal level $(\mathrm{dBm}) \geq-90$ \\
\hline & Best signal level $(\mathrm{dBm}) \geq-95$ \\
\hline & Best signal level $(\mathrm{dBm}) \geq-100$ \\
\hline & Best signal level $(\mathrm{dBm}) \geq-105$ \\
\hline
\end{tabular}

Table 2: Legend of signal strength coverage

\begin{tabular}{|l|l|}
\hline Colors & Legends \\
\hline & PDSCH/PDCCH total noise $(\mathrm{I}+\mathrm{N})(\mathrm{DL})(\mathrm{dBm}) \geq-50$ \\
\hline & PDSCH/PDCCH total noise $(\mathrm{I}+\mathrm{N})(\mathrm{DL})(\mathrm{dBm}) \geq-60$ \\
\hline & PDSCH/PDCCH total noise $(\mathrm{I}+\mathrm{N})(\mathrm{DL})(\mathrm{dBm}) \geq-70$ \\
\hline & PDSCH/PDCCH total noise $(\mathrm{I}+\mathrm{N})(\mathrm{DL})(\mathrm{dBm}) \geq-80$ \\
\hline & PDSCH/PDCCH total noise $(\mathrm{I}+\mathrm{N})(\mathrm{DL})(\mathrm{dBm}) \geq-90$ \\
\hline & PDSCH/PDCCH total noise $(\mathrm{I}+\mathrm{N})(\mathrm{DL})(\mathrm{dBm}) \geq-100$ \\
\hline & PDSCH/PDCCH total noise $(\mathrm{I}+\mathrm{N})(\mathrm{DL})(\mathrm{dBm}) \geq-110$ \\
\hline & PDSCH/PDCCH total noise $(\mathrm{I}+\mathrm{N})(\mathrm{DL})(\mathrm{dBm}) \geq-120$ \\
\hline
\end{tabular}

Table 3: Interference matrix

\begin{tabular}{|c|c|c|}
\hline $\begin{array}{l}\text { Interfered cell } \\
\text { Interfering cell }\end{array}$ & $\begin{array}{c}\text { Co-channel } \\
\text { interference probability }\end{array}$ & $\begin{array}{c}\text { Adjacent channel } \\
\text { interference probability }(\%\end{array}$ \\
\hline \multicolumn{3}{|l|}{ Site 12_2(0) } \\
\hline Site $12 \_1(0)$ & 0.38 & 0 \\
\hline Site $12 \_3(0)$ & 0.38 & 0 \\
\hline Site $9 \_-1(0)$ & 0.08 & 0 \\
\hline \multicolumn{3}{|l|}{ Site $1 \overline{4} \_3(0)$} \\
\hline Site $14 \_1(0)$ & 1.10 & 0 \\
\hline Site $14-2(0)$ & 1.10 & 0 \\
\hline \multicolumn{3}{|l|}{ Site $5 \overline{3}(0)$} \\
\hline Site $2 \_1(0)$ & 0.31 & 0 \\
\hline Site $22(0)$ & 0.31 & 0 \\
\hline Site $2-3(0)$ & 0.31 & 0 \\
\hline Site $5-1(0)$ & 0.89 & 0 \\
\hline Site $5-2(0)$ & 0.89 & 0 \\
\hline \multicolumn{3}{|l|}{ Site 6_3(0) } \\
\hline Site $1 \_1(0)$ & 0.96 & 0 \\
\hline Site 1_2(0) & 0.96 & 0 \\
\hline Site $1 \_3(0)$ & 0.96 & 0 \\
\hline Site $3 \_1(0)$ & 0.96 & 0 \\
\hline Site $6 \_1(0)$ & 0.96 & 0 \\
\hline Site $6 \_2(0)$ & 0.96 & 0 \\
\hline Site $9 \_1(0)$ & 1.09 & 0 \\
\hline \multicolumn{3}{|l|}{ Site $8-1(0)$} \\
\hline Site $1 \overline{4} \_3(0)$ & 0.23 & 0 \\
\hline Site $8 \_\overline{2}(0)$ & 0.70 & 0 \\
\hline Site $83(0)$ & 0.70 & 0 \\
\hline
\end{tabular}

co-channel interference probability and the adjacent channel interference probability. In this simulation, the main focus is on the concept of frequency reuse in Ayer 


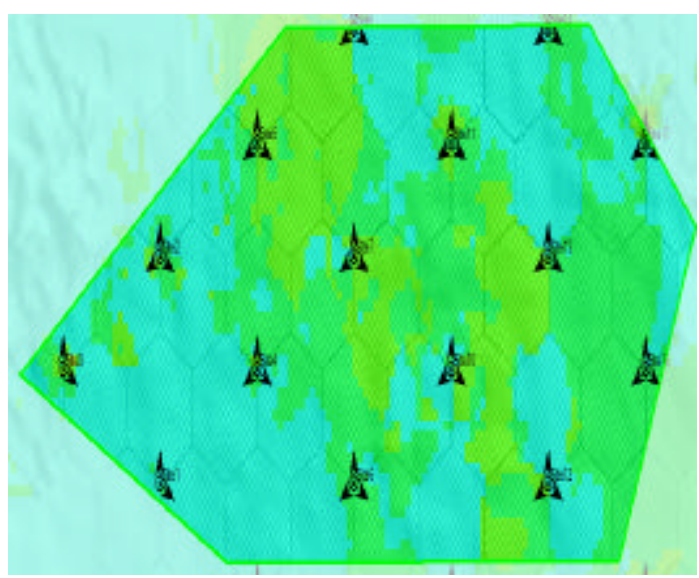

Fig. 7: Co-channel interference in downlink

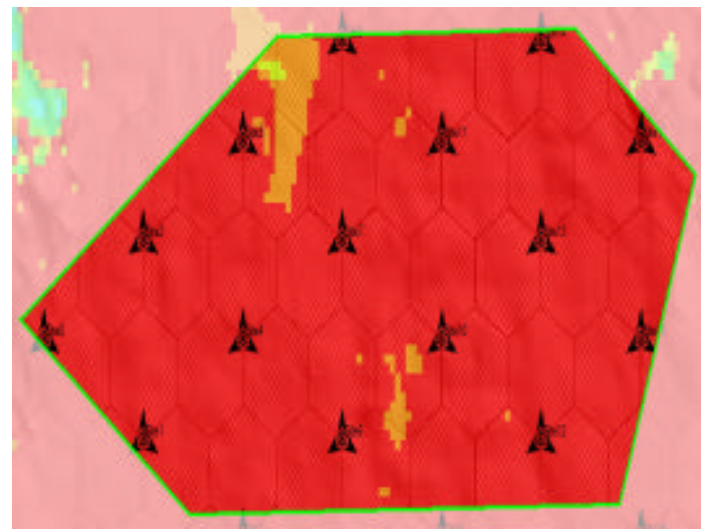

Fig. 8: Strong coverage of signal strength

Keroh location. From the results it can be seen that the signal strength is very strong as the coverage is majorly in red colour and a few portion in orange colour (Fig. 7) and the co-channel interferences is lower (Fig. 8 and 9) as the coverage area in green and blue colour is less. The interferences still exist in certain location is due to the frequency allocate in this software is very limited. There are only three different type of frequencies $(900,1800$ and $2100 \mathrm{MHz}$ ) are available with different degree and tilt. But in the frequency plan, the changes in degree and tilt does not influence its performance. There are few problems encounter in order to obtain a stronger signal strength with minimum co-channel interference in downlink. One of the problem is in identifying the parameters that need to be considered. One of the parameter is azimuth. When the azimuth of cell site is change, it results to blank spot and overlapping in between the adjacent cells. The blank spot is the area where there are no coverage. Secondly, when the frequency plan is calculated, the co-channel

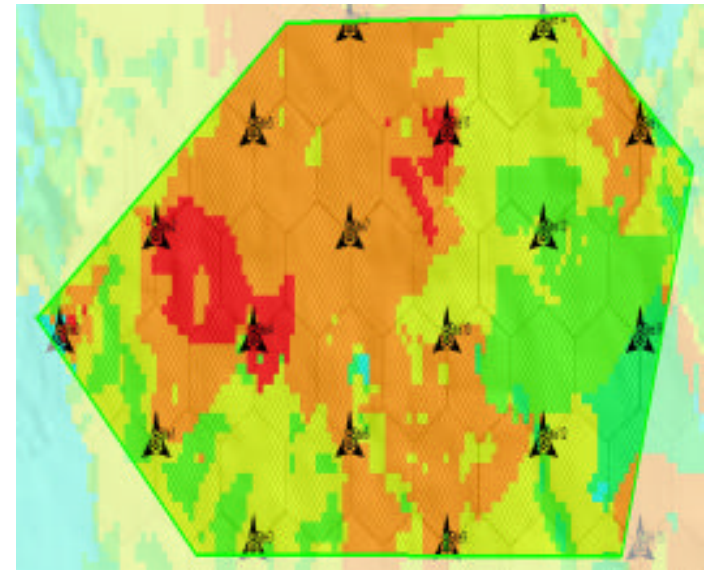

Fig. 9: Low co-channel interference

interference probability and adjacent channel probability is too high which is from $30 \%$ up to $100 \%$. According to International Telecommunication Union (ITU), the co-channel interference probability and adjacent channel probability should not be more than $20 \%$.

In order to increase the signal strength and reduce co-channel interference, other parameters such as antenna height and the power is considered. The Ayer Keroh area is not a flat land. For a peak area need an antenna with shorter height and a flat area need a longer antenna. If the height of antenna is same in all area as shown in Fig. 6, the signal strength is very weak as it will not be able to give a good coverage to all the area. So, the height of antenna should be varied according to the geographic area. The height of antenna is adjusted in the range of 30-90 m. The power of antenna is also change to increase the coverage. The default power in the software is $43 \mathrm{dBm}$ with this power it won't be able to give a good coverage for all the area. The value of power is varied in the range between 20 through $80 \mathrm{dBm}$ based on the geographical area. As the power increase, it will increase the coverage area. If the power is too high or same as cell neighbour, it might causes interferences in between cell neighbour.

\section{CONCLUSION}

Investigation for optimization of Fractional Frequency Reuse (FFR) in Orthogonal Frequency Division Multiple Access (OFDMA) network on selected area, Ayer Keroh, Melaka, Malaysia showed a significant improvement in term of co-channel interference and Received Signal Strength Indicator (RSSI).

Limited frequency allocation available in the simulation software cause the co-channel interference 
still exist, although, the values are considered very unsignificant. Improvement can be made by adding frequency resources and physical drive test to validate the simulation measurement result.

\section{ACKNOWLEDGEMENT}

The researchers would like to thank for the support given to this research by Ministry of Higher Education Malaysia and Universiti Teknikal Malaysia Melaka (UteM) under research Acculturation Grant Scheme (RAGS) No.RAGS/1/2015/ICT01/FTK/03/B00115 and the Grant PJP/2015/FTK(30A)/ S01455.

\section{REFERENCES}

Ali, S.H. and V.C. Leung, 2009. Dynamic frequency allocation in fractional frequency reused OFDMA networks. IEEE. Trans. Wirel. Commun., 8: 4286-4295.

Bilios, D., C. Bouras, V. Kokkinos, A. Papazois and G. Tseliou, 2012. A performance study of fractional frequency reuse in OFDMA networks. Proceedings of the 5th Joint IFIP Conference on Wireless and Mobile Networking (WMNC'12), September 19-21, 2012, IEEE, Bratislava, Slovakia, ISBN:978-1-4673-2993-4, pp: 38-43.

Bohge, M., J. Gross and A. Wolisz, 2009. Optimal power masking in soft frequency reuse based OFDMA networks. Proceedings of the 2009 European Conference on Wireless (EW'09), May 17-20, 2009, IEEE, Aalborg, Denmark, ISBN:978-1-4244-5935-3, pp: 162-166.
Bohge, M., J. Gross and A. Wolisz, 2010. Optimal soft frequency reuse and dynamic sub-carrier assignments in cellular OFDMA networks. Eur. Trans. Telecommun., 21: 704-713.

Chang, R.Y., Z. Tao, J. Zhang and C.C.J. Kuo, 2013. Dynamic Fractional Frequency Reuse (D-FFR) for multicell OFDMA networks using a graph framework. Wirel. Commun. Mobile Comput., 13: 12-27.

Efnusev, I., V. Stojanovksa and L. Dinov, 2010. Performance comparison of OFDMA and CDMA access technologies in broadband wireless communication networks. Proceedings of the 18th Conference on Telecommunications Forum (TELFOR'10), November 23-25, 2010, University of Belgrade, Serbia, Belgrade, pp: 384-387.

Ghaffar, R. and R. Knopp, 2010. Fractional frequency reuse and interference suppression for OFDMA networks. Proceedings of the 8th International Symposium on Modeling and Optimization in Mobile, Ad Hoc and Wireless Networks (WiOpt'10), May 31 June 4, 2010, IEEE, Avignon, France, ISBN:978-1 4244-7523-0, pp: 273-277.

Mitran, P. and C. Rosenberg, 2012. On fractional frequency reuse in imperfect cellular grids. Proceedings of the 2012 International Conference on Wireless Communication and Networking (WCNC'12), April 1-4, 2012, IEEE, Shanghai, China, ISBN:978-1-4673-0436-8, pp: 2967-2972.

Preethi, S.J. and K. Rajeswari, 2012. A survey on multiple access technique for mobile communication. Intl. J. Emerging Trends Technol. Comput. Sci., 1: $1-5$. 